\title{
Flexible and tenacious goal pursuit lead to improving well-being in an aging population: a ten-year cohort study
}

\author{
Rebecca E. Kelly, ${ }^{1}$ Alex M. Wood ${ }^{2}$ and Warren Mansell ${ }^{2}$ \\ ${ }^{1}$ Department of Psychology, Institute of Psychiatry, King's College London, London, UK \\ ${ }^{2}$ School of Psychological Sciences, University of Manchester, Manchester, UK
}

Background: Previous research has shown that tendencies to tenaciously pursue goals and flexibly adapt goals independently relate to well-being in adults in mid-to-late life, but research has not tested whether these tendencies interact. For example, tenacity may only predict well-being in combination with flexibility. This research tests whether these tendencies interact to predict changes in health-related outcomes.

Methods: A large cohort of people $(n=5,666)$, initially aged 55-56, completed measures of flexibility, tenacity, health-related outcomes (physical health, depression, hostility), as well as demographics. Participants provided follow-up data on all measures ten years later. Moderation analysis was used to test whether flexibility and tenacity interacted to predict changes in the health-related outcomes over the period.

Results: The interaction between tenacity and flexibility significantly predicted changes in depression, hostility, and physical ill-health symptoms over ten years, such that highly flexible and tenacious individuals experienced the largest decreases in symptoms of depression, hostility, and physical ill-health.

Conclusions: The interaction between flexibility and tenacity predicts greater well-being, such that one is most protective when an individual also scores highly on the other. The combination of flexibility and tenacity in the pursuit of personal goals may mean individuals can enjoy gains associated with goal pursuit without the detrimental effects of persevering in blocked goals.

Key words: goal pursuit, depression, hostility, ill-health, interaction

Tenacious goal pursuit (TGP) involves striving for goals with commitment and determination, engaging in assimilative processes to modify the environment in order to achieve one's goals. In contrast, flexible goal adjustment (FGA) involves pursuing goals with flexibility and disengaging from goals when necessary, engaging in accommodative processes to adjust to constraints and modify goals when there are obstacles (Brandtstadter and Rothermund, 2002). Previous research has suggested that individually both tenacious and flexible forms of goal pursuit are important for well-being (Brandtstadter and Rothermund, 2002). However, no previous research has tested whether the processes interact to predict well-being, for example, whether TGP is only related to wellbeing when combined with flexibility. This study addresses this gap in the existing literature.

Correspondence should be addressed to: Rebecca Kelly, Department of Psychology, Institute of Psychiatry, King's College London, 3rd Floor, Addiction Sciences Building, 4 Windsor Walk, Denmark Hill, London SE5 8AF, UK. Phone: +44 (0) 7736042 836. Email: rebecca.erin.kelly@gmail.com. Received 5 Dec 2011; revision requested 18 Jan 2012; revised version received 20 Jun 2012; accepted 3 Jul 2012. First published online 4 September 2012.
Converging evidence suggests that TGP may be beneficial to well-being. In both older and younger adult samples, there is a large body of research suggesting that striving for one's goals with persistence and effort and succeeding in personal goals is associated with greater well-being and less psychological distress. The pursuit of meaningful goals has been found to lead to coherence and a greater sense of control (Ryff and Keyes, 1995; Wood and Joseph, 2010), and the successful pursuit of goals has been consistently related to subjective well-being (e.g. Emmons, 1996). Further, it has been argued that when individuals are optimistic about the prospects of achieving their goals they might be likely to pursue their goals with more tenacity, effort, and persistence, and this may predict well-being (Segerstrom and Solberg-Nes, 2006). Such findings suggest that TGP may predict positive well-being and protect against psychological distress.

However, other research has suggested that in some situations TGP may be detrimental to well-being. Individuals who are highly optimistic and persistent in goal pursuit tend to experience 
more goal conflict (Segerstrom and Solberg-Nes, 2006), which can have negative consequences for well-being (Segerstrom and Solberg-Nes, 2006; Kelly et al., 2011). Further, while it may be adaptive to keep striving for a goal despite difficulty, "perserveration," the tendency to continue behavior even if it ceases to be effective or rewarding, and "perfectionism," the pursuit of high and rigid standards, have been found to be maladaptive (Serpell et al., 2009). Thus, it seems that TGP may be most beneficial to well-being when combined with a degree of flexibility. In such cases, the benefits of goal pursuit may be realized without the costs associated with continually pursuing ineffective or blocked courses of action.

The flexibility to change or discontinue behavior when it is ineffective, for example in the case of overwhelming obstacles to goals, may in itself be important in ensuring well-being. Being confronted with unattainable goals leads to distress and low well-being (Carver and Scheier, 1990), and failing to disengage from blocked goals is associated with rumination and the maintenance of unrealistic goals (Wrosch et al., 2003), while the ability to disengage from goals reduces the distress associated with repeated failure (Nesse, 2000). In line with this, Wrosch et al. (2003) stress the importance of the ability to disengage from blocked goals and identify new, important, valued goals in which to engage. This may be especially important in response to major stressful life events, for example, when individuals separate from a partner in midlife (Wrosch and Heckhausen, 1999), or discover they are unable to have children (Heckhausen et al., 2001).

Thus, the interaction between these two modes or styles of pursuing personal goals might be particularly relevant for the well-being of adults in mid-to-later life, and especially important when considering changes in well-being over time. The pursuit of personal projects and goals has been found to be an important determinant of wellbeing and positive affect in older adults (Lawton et al., 2002). However, when older individuals are highly committed to pursuing personal goals, it might be crucial that they are able to flexibly disengage from goals when obstacles arise. Multiple obstacles to the achievement of personal goals present themselves in later life, including decreases in functioning, financial difficulties, the loss of loved ones (Lerner et al., 2003), and reduced opportunities (Heckhausen and Schulz, 1995). Given that aging involves a series of stressful life events (Lerner et al., 2003), it seems particularly important that the tenacious pursuit of goals is matched with the flexibility to disengage when situationally appropriate.
To test this hypothesis, the present study investigates whether the interaction between FGA and TGP predicts changes in well-being over a ten-year period, in a sample of over 5,500 people initially aged 55-56 (Hauser and Sewell, 19572005). Well-being is a broad, multifaceted construct (Wood and Joseph, 2010), and this study focuses on the health-related outcomes of psychological distress, for example depressed mood, and physical ill-health symptoms. More specifically, this study focused on three specific indices of well-being: physical health, using a measure of physical illhealth symptoms; and measures of two dimensions of mood symptoms and psychological well-being, depression and hostility. These variables were selected as previous research has demonstrated the importance of goal-related constructs in predicting depression and other indices of psychological illbeing (e.g. Kelly et al., 2011). In addition, research suggests that depression and physical ill-health symptoms are common causes of suffering in older adult groups (e.g. Mecocci et al., 2004), and thus it was deemed important to investigate predictors of these variables in this study.

\section{Methods}

\section{Participants and procedure}

Participants completed the measures listed below as part of the Wisconsin project, which involved a sample of 10,317 men and women who graduated from Wisconsin high school in 1957. The average age at the start of the study was $21(S D=9.76)$. At multiple time points over the course of their adult lives, participants completed extensive telephone interviews and comprehensive questionnaire packs assessing various aspects of their lifestyle, occupation, financial circumstances, well-being, and health, and information was also collected from participants' family members. The present study focuses on data collected from questionnaires mailed to participants. At each collection wave, surviving participants who had participated in the previous wave were contacted to ask them to participate in the telephone interviews and to complete the mailout questionnaire packs. Two collection waves were of interest to this study. Time 1 refers to data collected from surviving participants from the original cohort who were contacted in 1993 and agreed to participate. Of these participants, 6,875 completed the mail questionnaires. Of this number, surviving participants were then followed up ten years later, between 2002 and 2004 (Time 2). Participants whose data were incomplete $(n=212)$ were excluded, yielding a final sample of 5,777 
Table 1. Sample demographics and baseline descriptive statistics

\begin{tabular}{lrr}
\hline & \multicolumn{1}{c}{$\mathrm{M}$} & \multicolumn{1}{c}{$\mathrm{SD}$} \\
Age & 59.14 & 0.49 \\
Total years of education & 13.71 & 2.32 \\
FGA - Flexibility & 17.66 & 3.71 \\
TGP - Tenacity & 17.87 & 3.82 \\
CESD - Depression & 16.37 & 15.64 \\
Hostility & 1.77 & 2.65 \\
Physical symptoms & 4.19 & 3.37 \\
\hline
\end{tabular}

CESD: Center for Epidemiological Studies Depression scale; FGA: flexible goal adjustment; TGP: tenacious goal pursuit.

individuals who completed measures at both time points. Participants were aged between 51-56 at Time 1 and $63-67$ at Time 2, and 54.7\% were female (see Table 1). Participants had 0-3 years of college education on average, and the majority of participants were married (85\%) and employed $(85.7 \%)$ at Time 1 . Detailed demographic data for the full sample are publically available (Hauser and Sewell, 1957-2005).

\section{Measures}

CENTER FOR EPIDEMIOlOgical STUdies DEPRESSION SCALE (CES-D; RADLOFF, 1977)

This is a self-report 20 -item measure of depression symptoms, for example, "how many days in the past week did you feel unhappy," on a scale of 0-7 days. The CES-D is one of the most widely used depression measures in research (Santor et al., 2006). The measure has excellent convergence with clinician ratings of depression (McDowell and Kristjansson, 1996), and Beekman et al. (1997) report $100 \%$ sensitivity and $80 \%$ specificity for depression in older adult populations. A single score is appropriate for the measure (Wood and Joseph, 2010) and so a sum was calculated if the respondent answered all of the items. For participants who did not answer all items but answered at least ten items, the mean of the individual's responses on the remaining items was imputed for missing values. Cronbach's $\alpha$ for the scale was $\alpha=0.90$ at Time 1 and $\alpha=0.88$ at Time 2 .

\section{HoSTILITY SCALE}

Three items from the State Trait Anger Expression Inventory (STAXI; Spielberger, 1988) were used as a brief assessment of anger and hostility symptoms. Participants were asked how many days in the past week $(0-7)$ they had experienced certain symptoms, for example, "how many days in the past week did you feel angry/hostile for several hours at a time?" Participants' responses to the three items
Table 2. Physical ill-health symptom list

\begin{aligned} \hline 1. & Aching muscles \\ 2. & Back pain/strain \\ 3. & Headaches \\ 4. & Chest pain \\ 5. & Constipation \\ 6. & Respiratory problems \\ 7. & Diarrhea \\ 8. & Urination problems \\ 9. & Dizziness/faintness \\ 10. & Excessive sweating \\ 11. & Fatigue/exhaustion \\ 12. & Trouble sleeping \\ 13. & Lack of energy \\ 14. & Stiff/swollen joints \\ 15. & Numbness \\ 16. & Visual problems \\ 17. & Skin problems \\ 18. & Shortness of breath \\ 19. & Ringing in ears \\ 20. & Nausea \\ 21. & Vomiting \\ 22. & Upset stomach \\ \hline & \end{aligned}

were summed, and if participants completed only two of the items the mean of their responses was imputed for the missing item. Cronbach's $\alpha$ for the scale was $\alpha=0.79$ at Time 1 , and $\alpha=0.81$ at Time 2 .

\section{MEASURE OF PHYSICAL SYMPTOMS}

Participants were asked whether they had experienced 22 symptoms over the past six months (e.g. "had headaches," see Table 2). Each item was coded 1 for a "yes" response and 0 for a "no" response. The summary score was the number of "yes" responses. The measure had excellent internal validity, with a Kuder-Richardson reliability coefficient $(K R-20)$ of 0.77 at Time 1 and 0.83 at Time 2 .

\section{Flexible Goal adjustment and}

Tenacious Goal Pursuit scales

(BRANDTSTADTER AND RENNER, 1990)

These are two 5-item scales, for example, "If I don't get something I want, I take it with patience" (FGA) and "I stick to my goals and projects even in the face of great adversity" (TGP). A full list of items is presented in Table 3. Participants rate each item on a scale of 1, "strongly agree," to 5, "strongly disagree," and then scores are reversed so that higher scores indicate high tenacity (TGP scale) and flexibility (FGA scale). Each scale was coded with a sum, and if at least three of the five items on each scale received a response the mean of valid items completed by the individual was imputed for 
Table 3. Short versions of the Brandstadter and Renner (1990) goal pursuit scales

\begin{tabular}{|c|c|}
\hline TGP items & $\begin{array}{l}\text { 1. Even when things seem hopeless, I keep } \\
\text { on fighting to reach my goals }(+) \\
\text { 2. I stick to my goals and projects even in } \\
\text { the face of great difficulties }(+) \\
\text { 3. The harder a goal is to achieve, the more } \\
\text { appeal it has to me }(+) \\
\text { 4. I can be very stubborn/obstinate in } \\
\text { pursuing my goals }(+) \\
\text { 5. To avoid disappointments, I do not set } \\
\text { my goals too high }(-)\end{array}$ \\
\hline FGA items & $\begin{array}{l}\text { 1. If I do not get something I want, I take it } \\
\text { with patience }(+) \\
\text { 2. It is very difficult for me to accept a } \\
\text { setback or defeat }(-) \\
\text { 3. I find it easy to see something positive } \\
\text { even in a serious mishap ( }+ \text { ) } \\
\text { 4. When everything seems to be going } \\
\text { wrong, I can usually find a bright side to } \\
\text { a situation (+) } \\
\text { 5. In general, I am not upset very long } \\
\text { about an opportunity passed up }(+)\end{array}$ \\
\hline
\end{tabular}

Note. $(+)$ indicates an item is positively scored $(-)$ indicates an item is reverse scored.

missing items. Both scales had very good internal validity, with $\alpha=0.68$ for FGA and $\alpha=0.73$ for TGP.

\section{Analysis}

Three, three-step hierarchical linear regressions were conducted to respectively predict changes in depression, hostility, and physical illness between the two time points. In each analysis, Time 2 levels of the outcome variable were regressed on Time 1 levels of the variable, along with the other predictors. Thus, the analysis predicts residualized changes in the outcome variable over time, or the variance in the Time 2 level of the variable that is not shared with Time 1 (Zapf et al., 1996).

Interactions between continuous variables are best tested using a multiple regression framework (Aiken and West, 1991) to avoid increased Type 1 and Type 2 error. A best practice multiple regression approach was therefore taken. A term representing the interaction between FGA and TGP was calculated by standardizing the two scores and multiplying the summed values. The key analysis involved simultaneously regressing the outcome on FGA, TGP, and the interaction, along with the covariates: age, gender, and education (years). A significant interaction suggests that the two predictors are non-independent and interact to predict the outcome (Aiken and West, 1991), such that the magnitude of the effect of one independent variable on the dependent variable varies as a function of a second independent, moderating variable. Significant interaction effects were subsequently graphed to view the nature of the interaction. The multiple regression moderation analysis (Aiken and West, 1991) used in this study allows the slope of the predictor to be plotted at different levels of the moderator variable. Using the regression prediction equation for high ( 1 standard deviation above the mean) and low (1 standard deviation below the mean) values of TGP, the graphs were created using Microsoft Excel plotting software. Simple slope analyses allowed for direct comparison of the effect at high and low levels.

\section{Results}

FGA and TGP scores at Time 1 were significantly and positively correlated, $r=0.53, p<0.001$.

\section{Depression symptoms}

A three-step, multiple regression analysis was performed to predict changes in depression symptoms from tenacity, flexibility, and their interaction (as well as covariates listed above) (see Table 4). The first step significantly predicted $\mathrm{T} 2$ depression $\left(F(4,5,777)=516.30, R^{2}=0.26\right.$, $p<0.001)$ from the covariates of age, education, gender, and T1 depression, each of which was a significant unique predictor. In the second step, TGP and FGA were entered, resulting in a significantly improved model $(\Delta F=-165.47$, $\left.\Delta R^{2}=0.004, p<0.001\right)$; both variables were unique negative predictors. However, entering the interaction in the third step further improved the model $\left(\Delta F=-45.81, \Delta R^{2}=0.003, p<0.001\right)$, with the interaction term reaching significance. This suggests that the relationship between TGP and changes in depression systematically differs depending on the level of flexibility (and equally, that the relationship between flexibility and changes in depression systematically depends on the level of TGP). Note that in moderation analysis the relevant effect size is not the change in $R^{2}$, which is always low in this type of analysis, but rather the relative difference in the size slope of the relationship between the predictor and the outcome and different levels of the mediator (Aiken and West, 1991). These relationships are depicted in Figure 1. The figures were constructed for low (1 standard deviation below the mean) and high (1 standard deviation above the mean) levels of flexibility and tenacity. Simple slope analyses were then conducted, which indicated that when flexibility is low, tenacity leads to relatively small decreases in depression $(r=-0.10)$. In contrast, 
Table 4. Regression analyses on changes in depression, hostility, and physical illness symptoms

\begin{tabular}{|c|c|c|c|c|c|c|c|c|}
\hline \multicolumn{3}{|c|}{ STEP 1} & \multicolumn{3}{|c|}{ STEP 2} & \multicolumn{3}{|c|}{ STEP 3} \\
\hline$B$ & $\beta$ & $\mathrm{P}$ & $B$ & $\beta$ & $\mathrm{P}$ & $B$ & $\beta$ & $\mathrm{P}$ \\
\hline
\end{tabular}

\section{Depression T2}

T1 symptoms

Age

Gender

Education

Flexibility

Tenacity

Interaction

$\begin{array}{rr}0.50 & 0.50 \\ -0.05 & -0.03 \\ 0.10 & 0.05 \\ -0.02 & -0.05\end{array}$

$\begin{array}{lrr}0.00 & 0.49 & 0.48 \\ \mathbf{0 . 0 3} & -\mathbf{0 . 0 5} & -\mathbf{0 . 0 3} \\ \mathbf{0 . 0 0} & \mathbf{0 . 1 0} & \mathbf{0 . 0 5} \\ \mathbf{0 . 0 0} & -\mathbf{0 . 0 2} & -\mathbf{0 . 0 5} \\ & -\mathbf{0 . 0 4} & -\mathbf{0 . 0 4} \\ & -\mathbf{0 . 0 3} & -\mathbf{0 . 0 3}\end{array}$

0.00

0.00
0.02

0.47

$-0.05$

0.00

0.10

0.00

$-0.02$

0.00

$-0.07$

0.02

$-0.06$

$-0.02$

0.47

$-0.03$

0.00

Hostility T2

T1 symptoms

Age

Gender

Education

Flexibility

Tenacity

Interaction

$\begin{array}{rr}\mathbf{0 . 3 8} & \mathbf{0 . 3 6} \\ 0.00 & 0.00 \\ 0.05 & 0.02 \\ -0.01 & -0.02\end{array}$

$\mathbf{0 . 0 0}$
0.87
0.08
0.08

$\begin{array}{rr}\mathbf{0 . 3 5} & \mathbf{0 . 3 5} \\ 0.00 & 0.00 \\ \mathbf{0 . 0 6} & \mathbf{0 . 0 3} \\ -0.01 & -0.02 \\ -\mathbf{0 . 0 9} & -\mathbf{0 . 0 7} \\ -0.01 & -0.01\end{array}$

$\mathbf{0 . 0 0}$
0.89
$\mathbf{0 . 0 3}$
0.07
$\mathbf{0 . 0 0}$
0.70

0.35

0.05

0.02

0.00

$-0.05 \quad 0.00$

$-0.07$

$\mathbf{0 . 0 0}$

$-0.06$

$\mathbf{0 . 0 0}$

Physical symptoms T2

\begin{tabular}{lrrrrrrrrr} 
T1 symptoms & $\mathbf{0 . 4 9}$ & $\mathbf{0 . 4 9}$ & $\mathbf{0 . 0 0}$ & $\mathbf{0 . 4 9}$ & $\mathbf{0 . 4 8}$ & $\mathbf{0 . 0 0}$ & $\mathbf{0 . 4 8}$ & $\mathbf{0 . 4 8}$ & $\mathbf{0 . 0 0}$ \\
Age & -0.02 & -0.02 & 0.08 & -0.04 & -0.02 & 0.08 & -0.04 & -0.02 & 0.08 \\
Gender & 0.01 & 0.01 & 0.28 & 0.03 & 0.02 & 0.18 & 0.03 & 0.02 & 0.15 \\
Education & -0.02 & -0.02 & 0.12 & -0.01 & -0.02 & 0.14 & -0.01 & -0.02 & 0.12 \\
Flexibility & & & & $-\mathbf{0 . 0 5}$ & $-\mathbf{0 . 0 4}$ & $\mathbf{0 . 0 0}$ & $-\mathbf{0 . 0 7}$ & $-\mathbf{0 . 0 7}$ & $\mathbf{0 . 0 0}$ \\
Tenacity & & & & -0.01 & -0.01 & 0.41 & $-\mathbf{0 . 0 4}$ & $-\mathbf{0 . 0 4}$ & $\mathbf{0 . 0 1}$ \\
Interaction & & & & & & & $-\mathbf{0 . 0 2}$ & $-\mathbf{0 . 0 7}$ & $\mathbf{0 . 0 0}$ \\
\hline
\end{tabular}

Note. T1 refers to Time 1 or baseline, T2 refers to Time 2 or follow-up. Significant effects $(p<0.05)$ in bold.
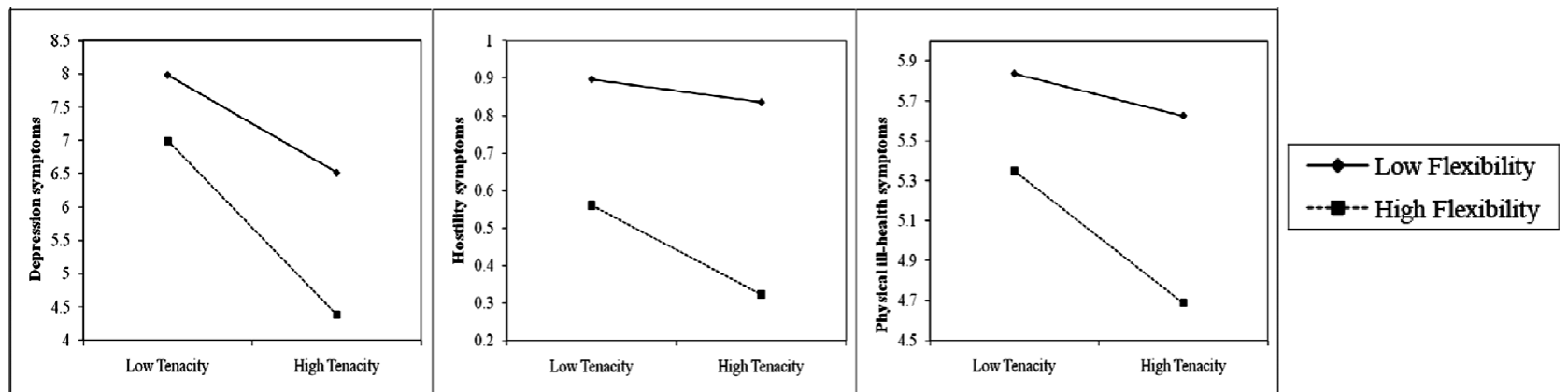

Figure 1. Graph depicting the effect of the interaction between TCP and FGA on symptoms of depression, hostility, and physical ill-health at Time 2, controlling for symptoms at Time 1 . Note. The high and low tenacity values are 1 standard deviation above and below the mean, respectively.

when flexibility is high, tenacity leads to larger decreases in depression $(r=-0.18)$. Individuals who scored 1 standard deviation above the mean on both the flexibility and tenacity measures reported experiencing approximately half as many symptoms of depression at Time 2 (when controlling for symptoms at Time 1) than those scoring 1 standard deviation below. The simple slope analysis revealed that while both gradients differed significantly from zero, the relationship between tenacity and changes in depression is $70 \%$ higher when flexibility is high compared to when it is low, and the significant interaction indicates that this difference is statistically significant. The same pattern of findings also emerged when comparing the effect of FGA on changes in depression symptoms at high and low levels of TGP.

\section{Hostility symptoms}

A three-step, multiple regression analysis was performed to predict changes in hostility symptoms 
from tenacity, flexibility, and their interaction (as well as covariates) (see Table 1). The first step significantly predicted $\mathrm{T} 2$ hostility ( $F$ $\left.(4,5,776)=216.69, R^{2}=0.14, p<0.001\right)$ from the covariates of age, education, gender, and $\mathrm{T} 1$ hostility symptoms. Only $\mathrm{T} 1$ hostility was a significant unique predictor. In the second step, TGP and FGA were entered, resulting in a significantly improved model $(\Delta F=-65.33$, $\left.\Delta R^{2}=0.006, p<0.001\right)$. FGA was a significant negative predictor, but TGP was not significant. In the third step, entering the interaction significantly improved fit $\left(\Delta F=-19.32, \Delta R^{2}=0.002\right.$, $p<0.001$ ), with the interaction term reaching significance. This suggests that the relationship between TGP and changes in hostility symptoms differs systematically depending on the level of FGA (and equally, that the association between flexibility and changes in hostility over time systematically depends on the level of tenacity). This interaction effect was then graphed for high and low values of TGP (Figure 1). Simple slope analyses of the graph revealed that when flexibility is low, tenacity leads to small decreases in hostility symptoms $(r=$ $-0.03)$. However, when flexibility is high, tenacity leads to considerably larger decreases in hostility $(r=-0.10)$. Again, the relevant effect size is the difference in the slope between the predicator and outcome at different levels of the moderator. Simple slope analysis revealed that the relationship between tenacity and changes in hostility symptoms is $91 \%$ higher when flexibility is high versus when flexibility is low. The significant interaction indicates that this difference is statistically significant. The same pattern was found when examining the effect of FGA on changes in hostility symptoms at high and low levels of TGP.

\section{Physical ill-health symptoms}

A three-step, multiple regression analysis was performed to predict changes in physical illness symptoms from tenacity, flexibility, and their interaction (as well as covariates) (see Table 1). The first step significantly predicted T2 symptoms $\left(F(4,5,777)=458.20, R^{2}=0.24, p<0.001\right)$ from the covariates of age, education, gender, and $\mathrm{T} 1$ illness symptoms. Only symptoms at $\mathrm{T} 1$ were a significant predictor in this step. In the second step, TGP and FGA were entered, resulting in a significantly improved model $(\Delta F=-148.46$, $\left.\Delta R^{2}=0.004, p<0.001\right)$. FGA was a significant negative predictor, but there was no significant effect of TGP on changes in illness symptoms. In the third step, entering the interaction in the third step significantly improved fit $(\Delta F=$ $\left.-30.38, \Delta R^{2}=0.003, p<0.001\right)$ and the interac- tion term was a significant predictor. This indicates that the relationship between TGP and changes in symptoms of physical ill-health systematically depends on individuals' level of flexibility (and equally, that the relationship between FGA and changes in illness symptoms also systematically depends on the level of tenacity). The interaction is graphed in Figure 1. Simple slope analyses, based on high and low levels of tenacity (1 standard deviation above and below the mean, respectively), were then conducted. When flexibility is low, tenacity leads to relatively small decreases in illness symptoms $(r=-0.04)$. However, when flexibility is high, tenacity leads to larger decreases in physical illness symptoms $(r=-0.13)$. Analysis of the simple slopes reveals that the association between tenacity and changes in illness symptoms when flexibility is high is $88 \%$ higher than when it is low. Again, the significant interaction indicates that this difference is statistically significant. Individuals who scored 1 standard deviation above the mean on both the flexibility and tenacity measures reported approximately one-third fewer physical illness symptoms at Time 2 (when controlling for symptoms at Time 1) than those scoring 1 standard deviation below. The same pattern was found when comparing the relationship between FGA and changes in symptoms of physical ill-health at high and low levels of TGP. (For brevity and clarity, the results are only reported for the relationship between TGP and symptoms at high (+1 SD) and low ( -1 SD) levels of FGA. The same pattern of findings was obtained for the effect of flexibility on symptoms at high and low levels of tenacity.)

\section{Discussion}

The interaction between TGP and FGA predicted symptoms of depression, hostility, and physical illhealth over time in an aging sample, over and above the effects of demographic variables, and controlling for baseline symptoms. Inspecting the interaction effects revealed that individuals who were high in both flexibility and tenacity experienced lower levels of depression, hostility, and ill-health symptoms. However, the impact of tenacity on changes in symptoms of ill-being was greater at high levels of flexibility, and for two of the three outcome measures did not reach significance at low levels of flexibility. This effect also applied in reverse; the effect of FGA on well-being was greater at high levels of tenacity. This is in line with the suggestion that TGP is most beneficial to wellbeing when combined with a higher degree of flexibility in goal adjustment. It is argued that this is because for individuals high in both tenacity and 
flexibility, they can enjoy the positive benefits of goal pursuit without experiencing the detrimental effects of persevering in ineffective or blocked courses of action.

If this study had not tested for an interaction effect and had instead considered main effects alone, the conclusion could have been drawn that flexibility was more important than tenacity in predicting changes in well-being, as the main effects suggested tenacity did not significantly independently predict on hostility or illness symptoms. However, in the presence of a significant interaction effect, main effects cannot be interpreted, and the interaction indicates that tenacity did effect changes in well-being symptoms, but only at high levels of flexibility. This finding calls into question the previous research that has suggested that FGA is more important than TGP in determining well-being in older adults (e.g. Brandtstadter et al., 1993; Bouerner, 2003), and highlights the importance of considering and testing for interaction effects between variables such as tenacity and flexibility. In fact, the results of this study indicate that both tenacity and flexibility are needed for positive outcomes across various healthrelated domains.

The findings of this study extend the existing literature and support the model proposed by Brandtstadter and Renner (1990), which suggests that both flexibility and tenacity in goal pursuit are important for well-being. However, the findings offer an important extension to the existing research and indicate that while tenacity and flexibility may represent distinct coping styles, in line with the proposal of Brandtstadter and Renner (1990), they do not seem to contribute independently to wellbeing, but rather they interact to predict well-being.

It is possible that flexibility and tenacity contribute differentially to positive and negative well-being, with increased tenacity contributing to increased positive well-being, and increased flexibility protecting against increases in negative well-being. This is concurrent with the suggestion of Carver and Scheier (1990) that goal regulation differentially impacts upon positive well-being and negative well-being. This possibility could be tested empirically in future research. This study focused on three facets of negative well-being as they are particular causes of suffering in the age group in question (e.g. Mecocci et al., 2004) and are outcomes known to be influenced by other goalrelated constructs (e.g. Kelly et al., 2011). However, well-being encompasses both subjective (SWB) and psychological well-being (PWB) (Joseph and Wood, 2010) and it is important to consider both positive and negative aspects of both SWB and PWB (Wood and Tarrier, 2010). Future research could investigate the importance of flexibility and tenacity in predicting aspects of SWB such as life satisfaction, positive affect, and negative affect, or aspects of PWB, for example, more existential constructs such as autonomy and personal growth. Further, while the current studies focus on how the interaction between tenacity and flexibility predicts changes in the well-being of older adults, future research needs to replicate the current study in different age groups, using a full range of well-being measures. It is hoped that the current findings will stimulate future research along these lines.

This study is limited by a reliance on selfreport measures of flexibility and tenacity and of the well-being measures. However, the depression measure has previously been found to converge with physician ratings of depression in older adult populations, with $100 \%$ sensitivity and $80 \%$ specificity (Beekman et al., 1997). Future research could extend these findings, perhaps using peer ratings of individuals' flexibility and tenacity in their goal pursuit. The study utilized a sample of adults in mid-to-later life, as the concepts of interest were proposed to be of particular relevance to well-being in aging populations. However, more research is needed to establish whether these findings generalize to other age groups.

The effect sizes of the moderation analyses were large, with the negative relationships between tenacity and the outcomes of hostility, depression, and physical ill-health being between $70 \%$ and $91 \%$ higher when flexibility was moderately high versus moderately low. The differences would be much higher again at high levels of flexibility (e.g. if we had examined the relationships at plus and minus two standard deviations of flexibility rather than one). Despite this, even at high levels of flexibility the negative relationships between tenacity and the outcomes were quite low $(r=-0.10$ to $r=0.18)$. However, the magnitude of these relationships needs to be considered in light of the very longterm (10 years) follow-up, where any prediction at all is notable, and in line with the effects commonly seen in the literature. Further, the basic theoretical contribution of the paper is to show that tenacity and flexibility interact to predict outcomes - this relationship was consistently and significantly shown and should, therefore, generalize to crosssectional or short-term follow-up studies, where basic relationships have previously been shown to be higher.

If these findings are replicated, then they may have implications for the understanding of psychological and physical ill-being. For individuals experiencing distress or low well-being, it might be helpful to consider the extent to which they are tenacious or flexible in their goal pursuit. 
There may be certain scenarios in later life when encouraging individuals to be more tenacious or flexible in their goal pursuit might be especially helpful and important. For example, in older adulthood, one major cause of low well-being and psychological distress is changes in physical health and functioning (e.g. Lerner et al., 2003). Professionals supporting individuals confronted with such changes, for example, the receipt of a diagnosis of a chronic or long-term health condition, could explore the personal goals that are important to this individual, helping them to either consider new ways to achieve certain goals, or encouraging them to adapt their goals and engage with different, more achievable goals. One study utilized a computerized intervention (Gould, 1989), which encouraged individuals to think about their goals, obstacles to goal achievement, and ways to achieve their goals, and obtained comparable outcomes to individuals who saw a professional therapist (Jacobs, 1995, cited by Bohart, 2000). Techniques which facilitate goal focus and reflection could also be incorporated in existing psychological treatments. For example, Kelly et al. (2012) argue that expressive writing interventions can help individuals consider their goals and reduce distress about ambivalence. Karoly (1993) proposes that a motivational perspective based on an understanding of individuals' dynamic and changing personal goals systems can aid assessment and the planning of psychological intervention. Clinicians may benefit from applying the concepts of flexibility and tenacity in goal pursuit when considering how to intervene to improve the well-being of older adults. Future research should also consider the wider relevance of these concepts to well-being.

\section{Conflict of interest}

None.

\section{Description of authors' roles}

All three authors contributed to the design of the study. The first author, Dr Kelly, undertook the statistical analyses, and wrote the first draft of the paper. All three authors contributed to and have approved the final paper.

\section{Acknowledgments}

The research was supported by the ESRC (RES060-25-0044). The first author's affiliation was School of Psychological Sciences, University of Manchester, UK, when this research was com- pleted. This research uses data from the Wisconsin Longitudinal Study (WLS) of the University of Wisconsin-Madison. Since 1991, the WLS has been supported principally by the National Institute on Aging (AG-9775, AG-21079, and AG-033285), with additional support from the Vilas Estate Trust, the National Science Foundation, the Spencer Foundation, and the Graduate School of the University of Wisconsin-Madison. A public use file of data from the Wisconsin Longitudinal Study is available from the Wisconsin Longitudinal Study, University of Wisconsin-Madison, 1180 Observatory Drive, Madison, Wisconsin 53706 and at http://www.ssc.wisc.edu/wlsresearch/data/. The opinions expressed herein are those of the authors.

\section{References}

Aiken, L. S. and West, S. G. (1991). Multiple Regression: Testing and Interpreting Interactions. Newbury Park, CA: Sage.

Beekman, A. T. F., Deeg, D. J. H., Limbeek, J. van, Braam, A. W., Vries, M. Z. De and Tilburg, W. van. (1997). Criterion validity of the Center for Epidemiologic Studies Depression scale (CES-D): results from a community-based sample of older adults in the Netherlands. Psychological Medicine, 27, 231-235.

Bohart, A. C. (2000). The client is the most important common factor: clients' self-healing capacities and psychotherapy. Fournal of Psychotherapy Integration, 10, 127-149.

Bouerner, K. (2003). Adaptation to disability among middle-aged and older adults: the role of assimilative and accommodative coping. Fournals of Gerontology, 59, 35-42.

Brandtstadter, J. and Renner, G. (1990). Tenacious goal pursuit and flexible goal adjustment: explication and age related analysis of assimilative and accommodative strategies of coping. Psychology and Aging, 5, 58-67.

Brandtstadter, J. and Rothermund, K. (2002). The life-course dynamics of goal pursuit and goal adjustment: a two-process framework. Developmental Review, 22, 117-150.

Brandtstadter, J., Wentura, D. and Greve, W. (1993). Adaptive resources of the aging self: outlines of an emergent perspective. International fournal of Behavioral Development, 16, 323-349.

Carver, C. S. and Scheier, M. F. (1990). Origins and functions of positive and negative affect: a control-process view. Psychological Review, 97, 19-35.

Emmons, R. A. (1996). Striving and feeling: personal goals and subjective well-being. In P. M. Gollwitzer and J. A. Bargh (eds.), The Psychology of Action: Linking Cognition and Motivation to Behaviour (pp. 313-337). New York: Guilford.

Gould, R. L. (1989). Therapeutic Learning Program [Computer software]. Santa Monica, CA: Interactive Health Systems.

Hauser, R. M. and Sewell, W. H. (principal investigators). Wisconsin Longitudinal Study (WLS) [graduates, siblings, and spouses]: 1957-2005, Version 12.26. Madison, WI: 
University of Wisconsin-Madison, WLS. Available at: http://www.ssc.wisc.edu/wlsresearch/documentation/; last accessed 17 August 2012.

Heckhausen, J. and Schulz, R. (1995). A life-span theory of control. Psychological Review, 102, 284-304.

Heckhausen, J., Wrosch, C. and Fleeson, W. (2001). Developmental regulation before and after passing a developmental deadline: the sample case of "biological clock" for child-bearing. Psychology and Aging, 16, 400-413.

Jacobs, M. (Chair). (1995). Computer psychotherapy: the direction of the future? Symposium presented at the Western Psychological Association Convention, Los Angeles.

Joseph, S. and Wood, A. M. (2010). Assessment of positive functioning in clinical psychology: theoretical and practical issues. Clinical Psychology Review, 30, 830-838.

Karoly, P. (1993). Goal systems: an organizing framework for clinical assessment and treatment planning. Psychological Assessment, 5, 273-280.

Kelly, R. E., Mansell, W. and Wood, A. M. (2011). Goal conflict and ambivalence interact to predict depression. Personality and Individual Differences, 50, 531-534.

Kelly, R. E., Wood, A. M., Shearman, K., Phillips, S. and Mansell, W. (2012). Encouraging acceptance of ambivalence using the expressive writing paradigm. Psychology and Psychotherapy: Theory Research and Practice, 85, 220-228.

Lawton, M. P., Moss, M. S., Winter, L. and Hoffman, C. (2002). Motivation in later life: personal projects and well-being. Psychology and Aging, 17, 539-547.

Lerner, R. M., Freedheim, D. K. and Weiner, I. B. (2003). Handbook of Psychology: Developmental Psychology. New Jersey: John Wiley and Sons Inc.

McDowell, I. and Kristjansson, E. (1996). Depression. In I. McDowell and C. Newell (eds.), Measuring Health: $A$ Guide to Rating Scales and Questionnaires (pp. 329-393). Oxford: Oxford University Press.

Mecocci, P., Cherubini, A., Mariani, E., Ruggiero, C. and Senin, U. (2004). Depression in the elderly: new concepts and therapeutic approaches. Aging Clinical and Experimental Research, 16, 176-189.
Nesse, R. M. (2000). Is depression an adaptation? Archives of General Psychiatry, 57, 14-20.

Radloff, L. S. (1977). The CES-D scale: a self-report depression scale for research in the general population. Applied Psychological Measurement, 1, 385-401.

Ryff, C. D. and Keyes, C. L. M. (1995). The structure of psychological well-being revisited. Fournal of Personality and Social Psychology, 69, 719-727.

Santor, D. A., Gregus, M. and Welch, A. (2006). Eight decades of measurement in depression. Measurement, 4, 135-155.

Segerstrom, S. C. and Solberg-Nes, L. (2006). When goals conflict but people prosper: the case of dispositional optimism. Fournal of Research in Personality, 40, 675-693.

Serpell, L., Waller, G., Fearon, P. and Meyer, C. (2009). The roles of persistence and perseveration in psychopathology. Behaviour Therapy, 40, 260-271.

Spielberger, C. D. (1988). Manual for the State Trait Anger Expression Inventory (STAXI). Odessa, FL: Psychological Assessment Resources.

Wood, A. M. and Joseph, S. (2010). The absence of positive psychological (eudemonic) well-being as a risk factor for depression: a ten year cohort study. Fournal of Affective Disorders, 122, 213-217.

Wood, A. M. and Tarrier, N. (2010). Positive clinical psychology: a new vision and strategy for integrated research and practice. Clinical Psychology Review, 30, 819-829.

Wrosch, C. and Heckhausen, J. (1999). Control processes before and after passing a developmental deadline: activation and deactivation of intimate relationship goals. Fournal of Personality and Social Psychology, 77, 415-427.

Wrosch, C., Scheier, M. F., Miller, G. E., Schultz, R. and Carver, C. S. (2003). Adaptive self-regulation of unattainable goals: goal disengagement, goal reengagement, and subjective well-being. Personality and Social Psychology Bulletin, 29, 1494-1508.

Zapf, D., Dormann, C. and Frese, M. (1996). Longitudinal studies in organizational stress research: a review of the literature with reference to methodological issues. Fournal of Occupational Health Psychology, 1, 145-169. 